EPiC Series in Engineering
Volume 3, 2018, Pages 162-172
HIC 2018. 13th International
Conference on Hydroinformatics

\title{
A semi-lagrangian scheme for advection-diffusion equation
}

\author{
Ersin Bahar', Gurhan Gurarslan ${ }^{1}$ \\ ${ }^{1}$ Pamukkale University, Department of Civil Engineering, Denizli, TR-20070, \\ Turkey
}

\section{Corresponding author: gurarslan@pau.edu.tr}

\begin{abstract}
This study proposes a semi-Lagrangian scheme for numerical simulation of advection-diffusion equation. The proposed method provides unconditional stability and highly accurate solutions even at large time steps. Another advantage of this method is that it requires a low computational time. Accuracy of the method is tested by a numerical application.
\end{abstract}

Keywords: Advection-diffusion; contaminant transport; method of characteristics; Saulyev scheme.

\section{Introduction}

The significant applications of advection-diffusion equation can be seen in fluid dynamics [1], heat transfer [2] and mass transfer [3]. The 3-D advection-diffusion equation without source is given by

$$
\begin{aligned}
\frac{\partial C}{\partial t}+U \frac{\partial C}{\partial x}+ & V \frac{\partial C}{\partial y}+W \frac{\partial C}{\partial z} \\
& =D_{x} \frac{\partial^{2} C}{\partial x^{2}}+D_{y} \frac{\partial^{2} C}{\partial y^{2}}+D_{z} \frac{\partial^{2} C}{\partial z^{2}}
\end{aligned}
$$

Where $\mathrm{t}$ is time, $\mathrm{x}, \mathrm{y}$ and $\mathrm{z}$ are spatial directions in cartesian coordinates, $\mathrm{C}$ is concentration of substance, $\mathrm{U}, \mathrm{V}$ and $\mathrm{W}$ are velocity components of flow in each direction and $\mathrm{D}$ represents the diffusivity coefficent in each direction.

In this paper, we consider one-dimensional advection-diffusion equation is given by: 


$$
\begin{aligned}
& \frac{\partial C}{\partial t}+U \frac{\partial C}{\partial x} \\
& =D_{x} \frac{\partial^{2} C}{\partial x^{2}}
\end{aligned}
$$

with $\mathrm{U}$ and $\mathrm{D}$ is constant, $0 \leq x \leq L$, and $0 \leq t \leq T$. $\mathrm{L}$ and $\mathrm{T}$ represent spatial and temporal boundaries of computational domain, respectively.

We denote the spatial and temporal step sizes by $\Delta x$ and $\Delta t$, respectively. Also Courant number, $\mathrm{Cr}$, is computed as $U \Delta t / \Delta x$ and the Peclet number, Pe, is obtained as $U \Delta x / D_{x}$.

The initial condition is $C(x, 0)=f(x)$ and boundary conditions are

$$
\begin{gathered}
C(0, t)=g_{0}(t) \\
0 \leq t \\
\leq T \\
C(L, t)=g_{1}(t), \\
0 \leq t \\
\leq T
\end{gathered}
$$

Where $f, g_{0}, g_{1}$ are known functions.

Accurate solution of equation (2) is very important for reducing existing pollutant concentrations or taking precautions by predicting pollution formation in water resources, which is the basic requirement of human beings. However, since this equation contains two different physical processes such as advection and diffusion, the precise numerical solution is quite difficult. To overcome this difficulty such as classical finite difference method [4], high-order finite element method [5], high-order finite difference methods [6,7], green element method [8], cubic and extended Bspline collocation methods [9-11], cubic, quartic and quintic B-spline differential quadrature methods [12,13], method of characteristics unified with splines [14-16], cubic trigonometric B-spline approach [17] Taylor collocation and Taylor-Galerkin methods [18] , Lattice Boltzmann method [19] have been developed. In addition, with the help of operator splitting methods, the appropriate methods for the physical processes of the problem can be combined.

In the scope of the study, equation (2) will be divided into two processes as the advection and diffusion by the operator splitting method. Method of characteristics with cubic spline interpolation (MOC-CS) and Saulyev scheme will be used for the 
solution of advection and diffusion processes, respectively. Though Saulyev scheme is an explicit method, it has the same order as the Crank-Nicolson scheme which is an implicit method. Saulyev scheme will shorten the computation time because it is explicit, but the precision of the solutions must be examined. For this purpose, the results obtained with Saulyev scheme will be compared with other methods in the literature and analytical solution of the problem.

\section{Numerical Discretization}

\subsection{Operator Splitting Approach}

Lie-Trotter operator splitting method is a first-order splitting method and solves problems sequentially. Its application to the equation (2) separates advection-diffusion equation into the two sub-problem such as advection and diffusion. Mathematical formulation of the application is as follows:

$$
\begin{gathered}
\frac{\partial \hat{C}_{1}}{\partial t}+U \frac{\partial \hat{C}_{1}}{\partial x}=0, \quad \hat{C}_{1}\left(t_{n}, x\right)=C\left(t_{n}, x\right), \\
t \quad\left[t_{n}, t_{n+1}\right] \\
\frac{\partial \hat{C}_{2}}{\partial t}=D_{x} \frac{\partial^{2} \hat{C}_{2}}{\partial x^{2}}, \quad \hat{C}_{2}\left(t_{n}, x\right)=\hat{C}_{1}\left(t_{n+1}, x\right), \\
t \in\left[t_{n}, t_{n+1}\right]
\end{gathered}
$$

Equation (5) and equation (6) represent advection and diffusion equations, respectively. These problems are solved consecutively, but are combined through the initial conditions. First, Equation (5) will be solved by the MOC-CS method at the time interval of $\Delta t$ using the initial condition of the general advection-diffusion problem and the result obtained from this will be used as the initial condition of the diffusion process. Then equation (6) will be solved in the time interval of $\Delta t$ with the help of the explicit Saulyev method and thus the solution of the advection-diffusion problem in the time interval of $\Delta t$ will be obtained.

\subsection{Numerical Discretization of Advection Part}

By multiplying both sides of the advection equation given in equation (5) by dt, this partial differential equation can be transformed into two ordinary differential equations as follows: 


$$
\begin{aligned}
& \frac{d \hat{C}_{1}}{d t} \\
& =0 \\
& \frac{d x}{d t} \\
& =U
\end{aligned}
$$

Thus, the equation (5) which is a partial differential equation can be solved as a simple ordinary differential equation along the line defined in the equation (8) in the plane $(x, t)$. As a result of equation (7), there is no change in the concentration along the characteristic line shown in Figure 1. In this case, the exact solution of equation (5) can be expressed as follows.

$$
\begin{aligned}
\hat{C}_{1}\left(x_{i+1}, t_{n+1}\right) & =\hat{C}_{1}\left(\hat{x}_{i}, t_{n}\right) \\
& =\hat{C}_{1}\left(x_{i+1}-\int_{t_{n}}^{t_{n+1}} U d t, t_{n}\right)
\end{aligned}
$$

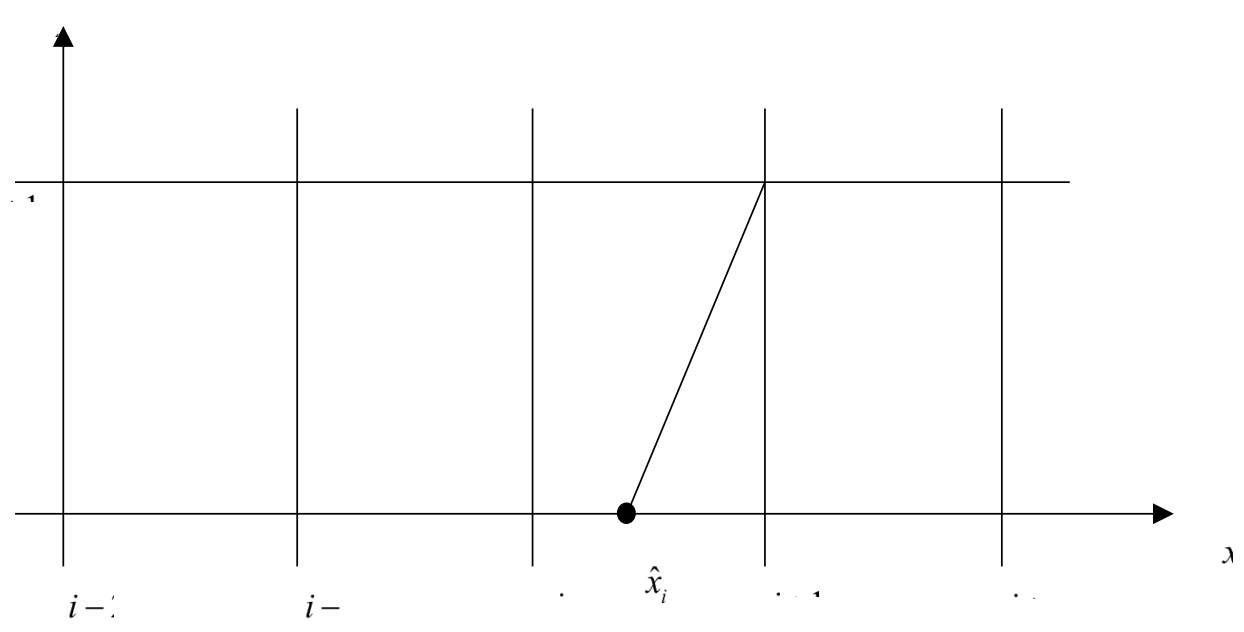

Figure 1 Finite difference grid and trajectory line of concentration in one-dimension

Since the coordinate of the point $\hat{x}_{i}$ is between the nodal points, interpolation must be made using the concentration values which are known from initial condition in the nodal points so that the concentration value at this point can be calculated. The only error encountered in solving the advection process is the interpolation. For this purpose, cubic spline polynomials with pretty low interpolation error will be used. Cubic spline polynomials can be written in the form 


$$
\begin{gathered}
C(x)=C_{i}+\alpha_{i}\left(x-x_{i}\right)+\beta_{i}\left(x-x_{i}\right)^{2}+\gamma_{i}\left(x-x_{i}\right)^{3} \\
x_{i} \leq x \leq x_{i+1}
\end{gathered}
$$

Where $C_{i}$ is the concentration value of at node $i$ and $\alpha_{i}, \beta_{i}, \gamma_{i}$ are polynomial coefficients determined from values at nodes. The detailed description about construction of the cubic spline polynomials and calculation of coefficients in the polynomials are given, for example, at [20].

After the polynomial is calculated in equation (10), the concentration values in all nodes at time $t_{n+1}$ can be calculated as follows.

$$
\begin{aligned}
\hat{C}_{1}\left(x_{i+1}, t_{n+1}\right) & =\hat{C}_{1}\left(x_{i}, t_{n}\right)+\alpha_{i}\left(\hat{x}_{i}-x_{i}\right)+\beta_{i}\left(\hat{x}_{i}-x_{i}\right)^{2} \\
& +\gamma_{i}\left(\hat{x}_{i}-x_{i}\right)^{3}
\end{aligned}
$$

As stated in equation (7), this method is time-independent and therefore does not have any stability condition. Solutions can be produced by selecting very large time steps.

\subsection{Numerical Discretization of Diffusion Part}

Although the Saulyev scheme is an explicit scheme, it uses values from the new time step. Thus, the quality of the solution is improving. There are two ways of solutions, from left to right and from right to left. The left to right version was used in the study. Spatial discretization of diffusion process via Saulyev scheme as follows:

$$
\begin{aligned}
& \left.\frac{\partial^{2} \hat{C}_{2}}{\partial x^{2}}\right|_{i, n} \\
& \approx \frac{\left.\frac{\partial \hat{C}_{2}}{\partial x}\right|_{i+1 / 2, n}-\left.\frac{\partial \hat{C}_{2}}{\partial x}\right|_{i-1 / 2, n}}{\Delta x}
\end{aligned}
$$

we can replace the left hand side derivate term at time level $n$ with derivative term at time level $n+1$ as the solution process goes from left to right. Similar modification can be done for right to left solution procedure.

$$
\begin{aligned}
& \left.\frac{\partial^{2} \hat{C}_{2}}{\partial x^{2}}\right|_{i, n} \\
& \approx \frac{\left.\frac{\partial \hat{C}_{2}}{\partial x}\right|_{i+1 / 2, n}-\left.\frac{\partial \hat{C}_{2}}{\partial x}\right|_{i-1 / 2, n+1}}{\Delta x},
\end{aligned}
$$

Also following approximations are used 


$$
\begin{aligned}
& \left.\frac{\partial \hat{C}_{2}}{\partial x}\right|_{i+1 / 2, n} \\
& \approx \frac{\left.\hat{C}_{2}\right|_{i+1, n}-\left.\hat{C}_{2}\right|_{i, n}}{\Delta x}
\end{aligned}
$$

$$
\begin{aligned}
& \left.\left.\frac{\partial \hat{C}_{2}}{\partial x}\right|_{i-1 / 2, n+1} \hat{C}_{2}\right|_{i-1, n+1} \\
& \approx \frac{\left.\hat{C}_{2}\right|_{i, n+1}-{ }^{2}}{\Delta x}
\end{aligned}
$$

$$
\begin{aligned}
& \left.\frac{\partial \hat{C}_{2}}{\partial t}\right|_{i, n} \\
& \approx \frac{\left.\hat{C}_{2}\right|_{i, n+1}-\left.\hat{C}_{2}\right|_{i, n}}{\Delta t}
\end{aligned}
$$

Combining these equations gives us the solution of diffusion equation using left to right Saulyev scheme as follows:

$$
=\frac{\left.\left.\theta \hat{C}_{2}\right|_{i, n+1}\right|_{i-1, n+1}+\left.(1-\theta) \hat{C}_{2}\right|_{i, n}+\left.\theta \hat{C}_{2}\right|_{i+1, n}}{(1+\theta)}
$$

where $\theta=\Delta t / \Delta x^{2}$.

At each unknown time level, $n+1$, at $i=1$ the term $\left.\hat{C}_{2}\right|_{0, n+1}$ is known from boundary conditions. Thus for $i>1$ the value of $\left.\hat{C}_{2}\right|_{i-1, n+1}$ is computed from equation (17), hence this method is explicit method.

\section{Numerical Applications}

In this section, we consider two one-dimensional advection-diffusion problems. One of them has a sharp gradient and the other one has smooth behavior. Obtained numerical results are compared with exact solution and the solutions of the other researchers in the literature. Many analyses have been done to test our numerical method's efficiency for a wide range of Courant numbers. Also error norms are compared and computed as follows: 
$L_{\infty}$

$=\max _{i} \mid C_{i}^{\text {exact }}$

$-C_{i}^{\text {numerical }}$

$L_{2}$

$=\sqrt{\sum_{i=1}^{M}\left|C_{i}^{\text {exact }}-C_{i}^{\text {numerical }}\right|^{2}}$

Example: Flow velocity and diffusion coefficient are taken as $U=0.01 \mathrm{~m} / \mathrm{s}$ and $D=$ $0.002 \mathrm{~m}^{2} / \mathrm{s}$ in this experiment. Length of the channel picked as $L=100 \mathrm{~m}$. Exact solution of this problem given as follows [21]:

$$
\begin{aligned}
C(x, t)=\frac{1}{2} \operatorname{erf} & f\left(\frac{x-U t}{\sqrt{4 D t}}\right) \\
& +\frac{1}{2} \exp \left(\frac{U x}{D}\right) \operatorname{erfc}\left(\frac{x+U t}{\sqrt{4 D t}}\right)
\end{aligned}
$$

Following boundary conditions are used:

$C(0, t)$

$=1$

$-D\left(\frac{\partial C}{\partial x}\right)(L, t)$

$=0$ 


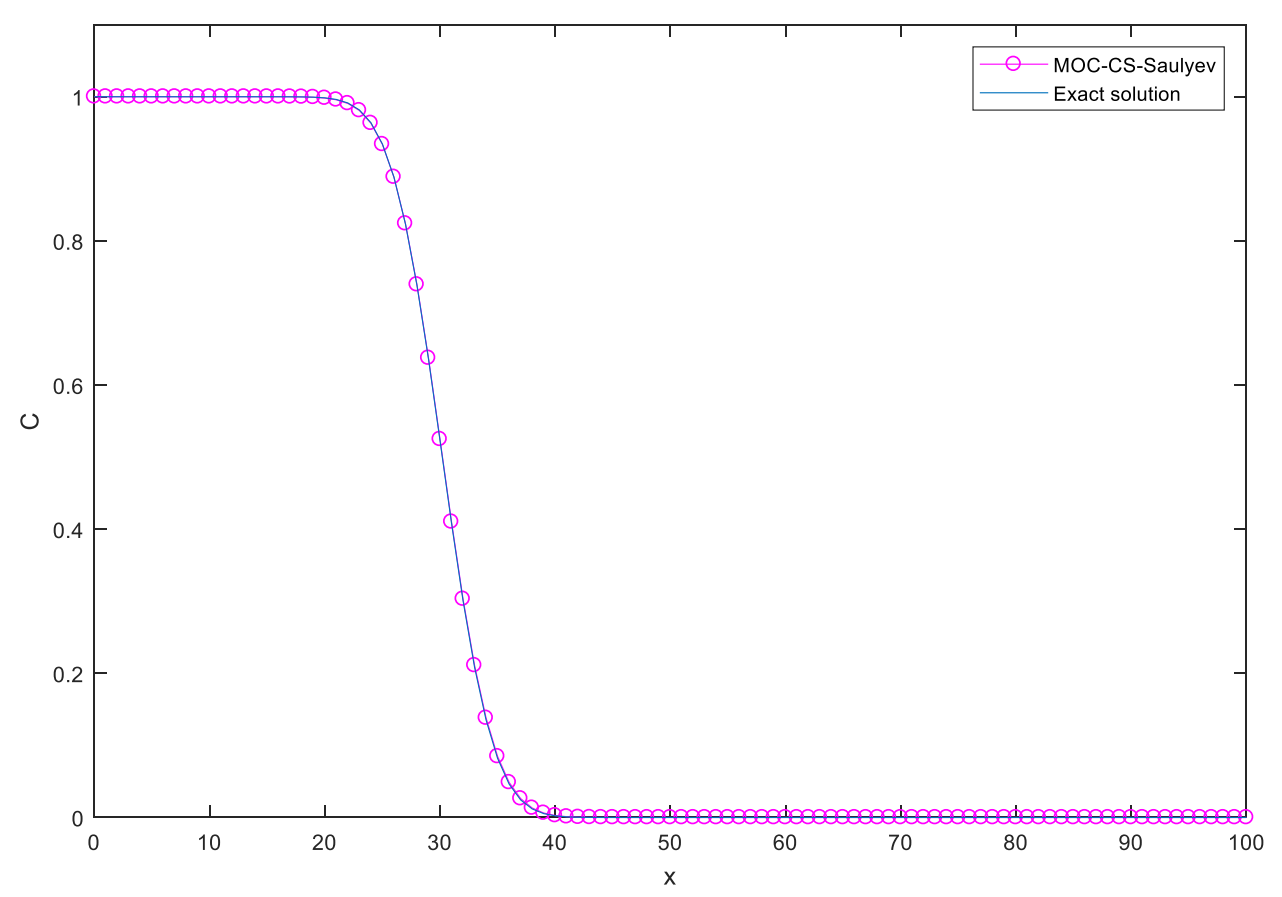

Figure 2 Comparison of the exact solution and the numerical solution obtained with MOCCS-Saulyev method for $\Delta x=1 \mathrm{~m}$ and $\Delta t=10 \mathrm{~s}$

In all calculations the spatial step size picked as $\Delta x=1 \mathrm{~m}$. Also maximum computational time taken as $3000 \mathrm{~s}$. As maximum computational time remain same in all analyzes, critical concentration values can be observed at from $18 \mathrm{~m}$ to $42 \mathrm{~m}$ (Figure 2). Also it should be taken to consideration that this problem is advection dominant $(P e=5)$ and has sharp shape. This makes the solution difficult for almost all numerical methods.

In Table 1, all concentration values obtained for the time interval of $\Delta t=10 \mathrm{~s}$. This makes Courant number equals to 0.1 . It clearly can be seen that MOC-CS-Saulyev scheme has closer results to exact solution compare to other numerical methods. This situation can also be recognized by looking at the error norms. It should be noted that even though MOC-CS-Saulyev has low order discretization compare to the sixth order compact finite difference it has smaller error norm values. 
Table 1 Comparison between numerical solutions and exact solution $(\Delta t=10 \mathrm{~s})$

\begin{tabular}{|c|c|c|c|c|c|}
\hline $\mathrm{x}(\mathrm{m})$ & $\begin{array}{c}{[7]} \\
\text { MC-CD6 }\end{array}$ & $\begin{array}{c}{[6]} \\
\text { RK4-CD6 }\end{array}$ & $\begin{array}{c}{[17]} \\
\text { CuTBSM }\end{array}$ & $\begin{array}{c}\text { MOC-CS } \\
\text { Saulyev }\end{array}$ & Exact \\
\hline 0 & 1.000 & 1.000 & 1.000 & 1.000 & 1.000 \\
\hline 18 & 1.000 & 1.000 & 1.000 & 1.000 & 1.000 \\
\hline 19 & 0.999 & 0.999 & 0.999 & 0.999 & 0.999 \\
\hline 20 & 0.998 & 0.998 & 0.998 & 0.998 & 0.998 \\
\hline 21 & 0.996 & 0.996 & 0.996 & 0.996 & 0.996 \\
\hline 22 & 0.991 & 0.992 & 0.991 & 0.991 & 0.991 \\
\hline 23 & 0.982 & 0.982 & 0.982 & 0.981 & 0.982 \\
\hline 24 & 0.965 & 0.965 & 0.963 & 0.964 & 0.964 \\
\hline 25 & 0.936 & 0.936 & 0.933 & 0.934 & 0.934 \\
\hline 26 & 0.891 & 0.891 & 0.885 & 0.889 & 0.889 \\
\hline 27 & 0.827 & 0.827 & 0.818 & 0.824 & 0.823 \\
\hline 28 & 0.743 & 0.743 & 0.732 & 0.739 & 0.738 \\
\hline 29 & 0.642 & 0.641 & 0.631 & 0.636 & 0.636 \\
\hline 30 & 0.529 & 0.528 & 0.517 & 0.524 & 0.523 \\
\hline 31 & 0.414 & 0.413 & 0.404 & 0.409 & 0.408 \\
\hline 32 & 0.306 & 0.306 & 0.298 & 0.302 & 0.301 \\
\hline 33 & 0.213 & 0.212 & 0.207 & 0.211 & 0.208 \\
\hline 34 & 0.138 & 0.138 & 0.134 & 0.138 & 0.135 \\
\hline 35 & 0.084 & 0.084 & 0.081 & 0.085 & 0.082 \\
\hline 36 & 0.048 & 0.048 & 0.045 & 0.049 & 0.046 \\
\hline 37 & 0.025 & 0.025 & 0.023 & 0.027 & 0.024 \\
\hline 38 & 0.012 & 0.012 & 0.011 & 0.014 & 0.012 \\
\hline 39 & 0.006 & 0.006 & 0.005 & 0.006 & 0.005 \\
\hline 40 & 0.002 & 0.002 & 0.002 & 0.003 & 0.002 \\
\hline 41 & 0.001 & 0.001 & 0.001 & 0.001 & 0.001 \\
\hline 42 & 0.000 & 0.000 & 0.000 & 0.000 & 0.000 \\
\hline $\mathrm{L}_{2}$ & 0.0148 & 0.0142 & - & 0.0071 & - \\
\hline $\mathrm{L}_{\infty}$ & 0.0060 & 0.0055 & - & 0.0031 & - \\
\hline
\end{tabular}

In Table 2, infinity error norms of MOC-CS-Saulyev, cubic B-spline and extended cubic B-spline collocation methods are compared for different time interval values. When Table 2 is examined it can be seen that MOC-CS-Saulyev scheme always has 
smaller error norm values except for extended cubic B-spline collocation method at the time interval of $\Delta t=1 \mathrm{~s}$.

Table 2 Comparison of $L_{\infty}$ error norms $(\Delta x=1 \mathrm{~m})$

\begin{tabular}{cccc}
\hline$\Delta \mathrm{t}(\mathrm{s})$ & $\begin{array}{c}{[11]} \\
\text { BSCM }\end{array}$ & $\begin{array}{c}{[11]} \\
\text { ECuBSCM }\end{array}$ & $\begin{array}{c}\text { MOC-CS } \\
\text { Saulyev }\end{array}$ \\
\hline 60 & 0.04330 & 0.04250 & 0.01235 \\
30 & 0.01962 & 0.01961 & 0.00635 \\
20 & 0.01270 & 0.01260 & 0.00471 \\
10 & 0.00685 & 0.00608 & 0.00314 \\
5 & 0.00409 & 0.00307 & 0.00243 \\
1 & 0.00224 & 0.00127 & 0.00193 \\
\hline
\end{tabular}

\section{Conclusions}

This paper deals with the advection-diffusion equation with the help of Lie-Trotter opeator splitting method. The problem splits into advection and diffusion processes. Each process solved by suitable methods for physical processes. Method of characteristics with cubic spline and Saulyev scheme is used for advection and diffusion, respectively. The effectiveness of method was tested using a onedimensional problem. The problem has a sharp gradient which is quite difficult to solve accurately. Obtained results are compared with exact solution and other researcher's solutions available in the literature.

\section{References}

[1] N. Kumar, "Unsteady flow against dispersion in finite porous media," Journal of Hydrology, vol. 63, no. 3-4, pp. 345-358, 1983.

[2] J. Isenberg and C. Gutfinger, "Heat transfer to a draining film," International Journal of Heat and Mass Transfer, vol. 16, no. 2, pp. 505-512, 1973.

[3] V. Guvanasen and R. E. Volker, "Numerical solution for solute transport in unconfined aquifers," International Journal for Numerical Methods in Fluids, vol. 3, no. 2, pp. 103-123, 1983.

[4] A. R. Appadu, "Numerical solution of the 1D advection-diffusion equation using standard and nonstandard finite difference schemes," Journal of Applied Mathematics, vol. 2013, Article ID 734374, 14 pages, 2013.

[5] H. S. Price, J. C. Cavendish and R. S. Varga, "Numerical methods of higher-order accuracy for diffusion-convection equations," Society of Petroleum Engineers, vol. 8, no. 3, pp. 293-303, 1968. 
[6] G. Gurarslan, H. Karahan, D. Alkaya, M. Sari and M. Yasar, "Numerical solution of advection-diffusion equation using a sixth-order compact finite difference method," Mathematical Problems in Engineering, vol. 2013, Article ID 672936, 7 pages, 2013.

[7] G. Gurarslan, "Accurate simulation of contaminant transport using high-order compact finite difference schemes," Journal of Applied Mathematics, vol. 2014, Article ID 396738, 8 pages, 2014.

[8] A. E. Taigbenu and O. O. Onyejekwe, "Transient 1D transport equation simulated by a mixed green element formulation," International Journal for Numerical Methods in Fluids, vol. 25, no. 4, pp. 437-454, 1997.

[9] R. C. Mittal and R. K. Jain, "Numerical solution of convection-diffusion equation using cubic B-splines collocation methods with Neumann's boundary conditions," International Journal of Applied Mathematics and Computation, vol. 4, no. 2, pp. 115127, 2012.

[10] J. Goh, A. A. Majid and A. I. M. Ismail, "Cubic B-spline collocation method for onedimensional heat and advection-diffusion equations," Journal of Applied Mathematics, vol. 2012, Article ID 458701, 8 pages, 2012.

[11] D. Irk, I. Dag and M. Tombul, "Extended cubic B-spline solution of the advectiondiffusion equation," KSCE Journal of Civil Engineering, vol. 19, no. 4, pp. 929-934, 2015.

[12] A. Korkmaz and I. Dag, "Cubic B-spline differential quadrature methods for the advection-diffusion equation," International Journal of Numerical Methods for Heat and Fluid Flow, vol. 22, no. 8, pp. 1021-1036, 2012.

[13] A. Korkmaz and I. Dag, "Quartic and quintic B-spline collocation methods for advection-diffusion equation," Applied Mathematics and Computation, vol. 274, pp. 208-219, 2016.

[14] F. M. Holly Jr. and A. Preissmann, "Accurate calculation of transport in two dimensions," Journal of Hydraulic Division, vol. 103, no. 11, pp. 1259-1277, 1977.

[15] T.-L. Tsai, J.-C. Yang and L.-H. Huang, "Characteristics method using cubic-spline interpolation for advection-diffusion equation," Journal of Hydraulic Engineering, vol. 130, no. 6, pp. 580-585, 2004.

[16] T.-L. Tsai, S.-W. Chiang and J.-C. Yang, "Examination of characteristics method with cubic interpolation for advection-diffusion equation," Computers and Fluids, vol. 35, no. 10, pp. 1217-1227, 2006.

[17] T. Nazir, M. Abbas, A. I. M. Ismail, A. A. Majid and A. Rashid, "The numerical solution of advection-diffusion problems using new cubic trigonometric B-spline approach," Applied Mathematical Modelling, vol. 40, pp. 4586-4611, 2016.

[18] I. Dag, A. Canivar and A. Sahin, "Taylor-Galerkin method for advection-diffusion equation," Kybernetes, vol. 40, no. 5/6, pp. 762-777, 2011.

[19] J. G. Zhou, "A lattice Boltzmann method for solute transport," International Journal for Numerical Methods in Fluids, vol. 61, pp. 848-863, 2009.

[20] R. S. Esfandiari, Numerical Methods for Engineers and Scientists Using MATLAB, New York: Taylor and Francis Group, 2013. 